\title{
Implications of cohesive strength in asteroid interiors and surfaces and its measurement
}

\author{
Daniel J. Scheeres ${ }^{*}$ and Paul Sánchez
}

\begin{abstract}
Recent observations and theory have indicated that rubble pile asteroids may have a small, but finite, level of tensile strength, allowing them to spin above their spin deformation limit as defined in Holsapple (Icarus 205:430-442, 2010). In Sánchez and Scheeres (Meteorit Planet Sci 49:788-811, 2014), a theory for how such strength could be present in rubble pile asteroids was presented, relying on weak van der Waals forces between fine particulate material in asteroid regolith and in their interiors. The implications of this theory are evaluated and related to the surface strength of regolith and global strength of a rubble pile body. Proposed techniques to measure the strength of regolith using cratering theory are reviewed, as are constraints placed on the global strength of rubble pile asteroids from astronomical observations. Specific examples applied to the Hayabusa2 cratering experiment at its target asteroid are given.
\end{abstract}

Keywords: Asteroid regolith, Cohesion, Rubble pile asteroids, Asteroid fission

\section{Introduction}

This paper reviews and explores the possible existence of rubble pile asteroids having a small, but finite, level of tensile strength, allowing them to spin above their spin deformation limit as defined by Holsapple (2010). The existence of a weak strength in rubble piles has significant implications for their physical evolution and should control the formation of binaries, small tumbling asteroids, and the eventual disaggregation of small, cohesive asteroids. The theory that rubble pile bodies should be controlled by cohesive forces was first introduced and discussed in (Asphaug 2009) and (Scheeres et al. 2010). Motivated by these basic ideas and the observed structure and size distribution of grains for asteroid Itokawa, in Sánchez and Scheeres (2014), these ideas were further developed into a theory for how such strength could be present in rubble pile asteroids, relying on weak van der Waals forces between fine particulate material in asteroid regolith and in their interiors. The upcoming sample return missions Hayabusa2 (Yoshikawa et al. 2014) and OSIRIS-REx (Lauretta et al. 2017) will provide unprecedented insight into the strength of an asteroid's surface

\footnotetext{
* Correspondence: scheeres@colorado.edu

Colorado Center for Astrodynamics Research, The University of Colorado, Boulder, CO 80309, USA
}

regolith covering, which will in turn provide insight into the genesis of tensile strength within rubble pile bodies.

This paper discusses several implications of this theory for the surface properties and sampling of asteroid regolith, and the global implications for the overall evolution of a cohesive rubble pile asteroid. Predictions, interpretations of astronomical observations, and methods for measuring cohesion are given. The paper is structured as follows. First, the general Sánchez and Scheeres (2014) theory of cohesion within regolith is reviewed. Then, the implications of the predicted levels of strength for the properties and sampling of surface regolith are discussed and the implications for the evolution of rubble pile bodies-noting that these elements are connected. Then, an approach for the measurement of cohesive strength in surface regolith is proposed and developed, including predictions for the upcoming Hayabusa2 mission and other proposed planetary science missions. This is followed by a review of several observed asteroids that exhibit levels of global strength commensurate with the theory, along with specific predictions. Finally, a discussion of the results is provided followed by a conclusion section. 


\section{Methods}

\section{The theory of cohesion in rubble pile asteroids}

In Sánchez and Scheeres (2014), a theory for how cohesion can arise in rubble pile asteroids was developed and applied to solar system asteroidal bodies. The approach essentially rests on the documented properties of cohesive powders in terrestrial environments and assumed cohesive properties of fine regolith as measured on the lunar surface.

The theory rests on a few observations motivated by observations of the asteroid Itokawa. First is the size distribution of components on the surface of Itokawa from decameters to decimeters and smaller (Michikami et al. 2008) and in the sample that was collected from $100 \mu \mathrm{m}$ down to microns (Tschumiyama et al. 2011). Both of these were approximately volume conserving, meaning that the cumulative size distributions of both collections were on the order of $1 / d^{3}$ where $d$ is the diameter of the grain. While the complete size distribution from decameters to microns is not known-and on the surface seems to be truncated at millimeters (although this may be driven by surface dust levitation effects (Wang et al. 2016)) - the interior is likely to have a much larger number of fine grains. Due to this, it can be hypothesized that the surfaces of interior boulders and grains of this asteroid are likely covered with finer regolith grains and, due to the numbers implied by the size distributions, that it is likely that these finer regolith grains are mutually connected forming a weak "matrix" of material.

If this is true, we can estimate the strength of such a matrix using traditional theory for dry cohesion between powders. Using measured properties of lunar regolith and accounting for correction factors related to porosity and number of contacts between grains, Sánchez and Scheeres (2014) developed a simple formula for the strength of a weakly packed regolith matrix $\sigma_{\mathrm{c}}=3 \times 10^{-4} / \bar{d}$ Pa where $\bar{d}$ is the mean diameter of the grains in meters (in a $1 / d^{3}$ size distribution, this will be just twice the smallest particle size counted in the distribution). This formula represents a simple and well-known fact that the strength of a powder will increase with a decrease in the size of its constituents and explains why flour acts as a cohesive powder while granular sugar does not. The formula depends on a number of parameters that can change from body to body, including the constituent minerals and the packing (or porosity) of the material. For a mean grain size of $1 \mu \mathrm{m}$, the formula predicts a strength of $300 \mathrm{~Pa}$; for a mean grain size of $10 \mu \mathrm{m}, 30 \mathrm{~Pa}$; and for $100 \mu \mathrm{m}, 3 \mathrm{~Pa}$. While these strengths seem vanishingly small, they provide enough strength to change the geophysical evolution of rubble pile bodies.

For a larger grain embedded within such a matrix, we note that the cohesive forces between the fine particles and the larger grain will be a bit stronger than the cohesive forces between the more similar-sized particles, meaning that the larger grain will be held within the matrix with a strength at least of the matrix. The net force acting on a larger grain of diameter $D$ embedded within a matrix of strength $\sigma_{\mathrm{c}}$ is calculated in Sánchez and Scheeres (2014) to be $\sim(\pi / 2) D^{2} \sigma_{\mathrm{c}}$.

For characterizing the relative effect of cohesion in terrestrial powders, the ratio of the cohesive force acting on a grain to its weight is used as a non-dimensional measure of the strength, commonly called the bond number (Castellanos 2005, Perko et al. 2001, Scheeres et al. 2010). In the theory of cohesive powders, distinct changes in material behavior are seen once the bond number exceeds unity, in general, with the material acting more and more as a solid as bond number increases. On the surfaces of asteroids, the net attraction (due to gravitational attraction and centripetal acceleration) is often quite small, on the order of milli- to micro-G's $\left(1 \times 10^{-2} \rightarrow 1 \times 10^{-5} \mathrm{~m} / \mathrm{s}^{2}\right)$, meaning that the weight of a grain will be quite small as well. Computing the bond number of a grain on the surface of an asteroid with net acceleration $g$ and density $\rho$ is then $B=\frac{3 \sigma_{c}}{8 \rho D g}$.

Using this formula, typical bond numbers of grains within an asteroid can be computed. We will see that they tend to be quite large, even for boulders. Consider a density of $\rho=2000 \mathrm{~kg} / \mathrm{m}^{3}$ and grains of size $1 \mathrm{~mm}$ to $10 \mathrm{~m}$, accelerations from $1 \mathrm{~m}-\mathrm{G}$ to 1 micro-G, and regolith strength from 1 to $100 \mathrm{~Pa}$. For a $1 \mathrm{~mm}$-sized grain ,the lowest bond number (weakest cohesion and greatest gravity) is 20 (at $1 \mathrm{~m}-\mathrm{G}$ ) and increases up to $2 \times 10^{6}$ for the strongest cohesion in the weakest gravity field. A $1 \mathrm{~m}$-sized boulder will similarly vary from 0.02 to 2000 and a $10 \mathrm{~m}$ boulder from 0.002 to 200 . Thus, even for decameter-sized boulders within an asteroid, they can be at large enough bond numbers to act, in aggregate, as a solid. These computations highlight the significance that even weak cohesion can have on the behavior of rubble pile bodies. They also show interesting transition values, which could be used to better understand and interpret the global behavior of a rubble pile. Specifically, for our assumed density, a $10 \mathrm{~m}$ boulder will have a unity bond number in micro-G for a regolith strength of $0.5 \mathrm{~Pa}$ and in milli-G for a regolith strength of $500 \mathrm{~Pa}$. For a metersized boulder, the unity bond numbers range from 0.05 to $50 \mathrm{~Pa}$. These values are important, as the behavior of these larger boulders is probably important for the geophysical evolution of rubble piles when they reach rapid spin rates.

The final step in this discussion is to review what the spin rate for the global failure of a cohesive rubble pile body would be-global implying that the body will fission into two or more components. Again in Sánchez and Scheeres (2014), an approximate relationship is found for the global failure spin rate of a rubble pile body, $\omega_{\mathrm{F}}$ assuming a Drucker-Prager failure condition, a conservative 
friction angle of $90^{\circ}$, an ideal shape approximated as a sphere, and with a given level of cohesive strength to be

$$
\omega_{\mathrm{F}}^{2}=\omega_{\mathrm{C}}^{2}+\sigma_{\mathrm{c}} /\left(\rho R^{2}\right)
$$

where $\omega_{\mathrm{C}}^{2}=4 \pi G \rho / 3$ is the spin limit at which surface particles are lofted off (and approximately at which a cohesionless body will undergo deformation (Holsapple 2010, Sánchez and Scheeres 2012)) and is only a function of density for a spherical body, $G$ is the gravitational constant, $\rho$ is the bulk density, and $R$ is the body radius. The formula makes assumptions on friction angle for the rubble pile, shape of the body, and other factors that can change the second term (called the strength spin rate) by a factor of a few-however, it still captures the qualitative nature. We note that for a large or weak body, the cohesion term will be small. Conversely, for a small or strong body, the cohesive term can dominate. We define an analogous "global" bond number as the ratio of the cohesive strength term over the failure condition, defined as $\Gamma=\sqrt{\frac{3 \sigma_{c}}{4 \pi G}} \frac{1}{\rho R}$. The failure condition is then $\omega_{\mathrm{F}}=\omega_{\mathrm{C}} \sqrt{1+\Gamma^{2}}$. For $\Gamma>1$, the cohesive strength dominates over the gravitational term and vice-versa for $\Gamma<1$. This is discussed in more detail below.

\section{Results}

\section{Implications of cohesion in asteroids}

If one assumes that the regolith, or at least interstitial regolith beneath the surface of an asteroid, is cohesive, then this has implications in our models for how space vehicles will interact with the surface and for the physical evolution of these bodies when subject to non-gravitational effects that cause their spin rates to increase over time. The following subsections review and apply recent work that has explored both of these topics.

\section{Implications for surface sampling}

In micro-gravity environments, the strength of surface regolith that has friction but lacks any cohesive forces behaves in a fluidic way, due to the low weight of the objects and the resulting weak frictional forces between materials within the regolith (Bierhaus et al. 2018). A massive object that intrudes into such a distribution is able to push the material out of the way, creating a large crater consistent with simple theory (reviewed below). As cohesive strength is added to the regolith, indicative of a finer fraction of regolith that supplies additional strength as reviewed above, the penetration of a sampling device into the regolith becomes limited.

In Figs. 1 and 2, we show the results of simulations of the intrusion of the OSIRIS-REx sampling arm into regolith modeled in a micro-gravity environment, chosen to mimic the expected surface properties of the OSIRIS-REx mission. The regolith is modeled as a size-diapered set of spherical grains with a diameter ranging from 0.8 to $1.2 \mathrm{~cm}$, the density of the grains is $2500 \mathrm{~kg} / \mathrm{m}^{3}$, porosity of $36 \%$ (giving a bulk density of 1600), and a friction angle of $35^{\circ}$. The surface acceleration is $10^{-5} \mathrm{~m} / \mathrm{s}^{2}$ due to a combination of gravity and centripetal acceleration. The total mass of the spacecraft $(\mathrm{S} / \mathrm{C})$ is taken to be $1300 \mathrm{~kg}$, and it impacts the surface at $10 \mathrm{~cm} / \mathrm{s}$. The sampling head is connected to the rest of the S/C via the long arm shown. The arm itself has a constant force spring that is activated when the force goes beyond $60 \mathrm{~N}$, isolating the $\mathrm{S} / \mathrm{C}$ bus from the ground force. The strength values modeled are 50, 150, and $300 \mathrm{~Pa}$. For the weakest regolith, the spring is never activated, meaning that the regolith never provides this level of force. This can be estimated by considering the effective force that the regolith can provide due to its cohesive strength. The area of the sampling head when fully engaged with the regolith is approximately $0.3 \mathrm{~m}^{2}$. Thus, the surface force that the regolith can provide starts at $15 \mathrm{~N}$, much weaker than the spring. At this level of force, the $\mathrm{S} / \mathrm{C}$ will slow and stop over approximately $8 \mathrm{~s}$, which is consistent with the simulation. Here, we note that the regolith is excavated in a cratering-like event, meaning that the subsurface regolith is not able to develop a strength cone and provide a larger force. For the $150 \mathrm{~Pa}$ case, we see the assembly at its lowest point. In this case, the initial force from the regolith is just under $50 \mathrm{~N}$; however, the regolith is now compressed and does not excavate, meaning that a strength cone forms and rapidly pushes against the $\mathrm{S} / \mathrm{C}$ with a force greater than $60 \mathrm{~N}$, activating the spring. Here, the crater size is a bit larger than the head, implying the importance of geometrical effects. The $300 \mathrm{~Pa}$ case provides an initial force of up to $95 \mathrm{~N}$, immediately engaging the spring and essentially stopping the assembly at the surface. Larger and smaller values of strength have a similar result as the end cases, showing that the surface response changes rapidly over a relatively narrow range of regolith strength.

\section{Implications for rubble pile fission}

If a rubble pile has interstitial regolith between its components, including a fine fraction small enough to generate strength, then the overall body can start to behave as a weak solid, with cohesive strength actively competing with gravitational attraction. Using the conservative strength model from Eq. 1, we can quantify the relative strength of the body with the "global" bond number for a rubble pile defined earlier, $\Gamma$.

When $\Gamma=1$, the strength spin rate term in Eq. 1 equals the spin rate $\omega_{\mathrm{C}}$, with the failure spin rate increasing to $\sqrt{2} \omega_{\mathrm{C}}$. This spin rate also marks an important transition on how the rubble pile will evolve once the failure spin rate is reached. For $\Gamma<1$, any components that separate from the body will in general be in bound orbits relative to each other, enabling the system components to 


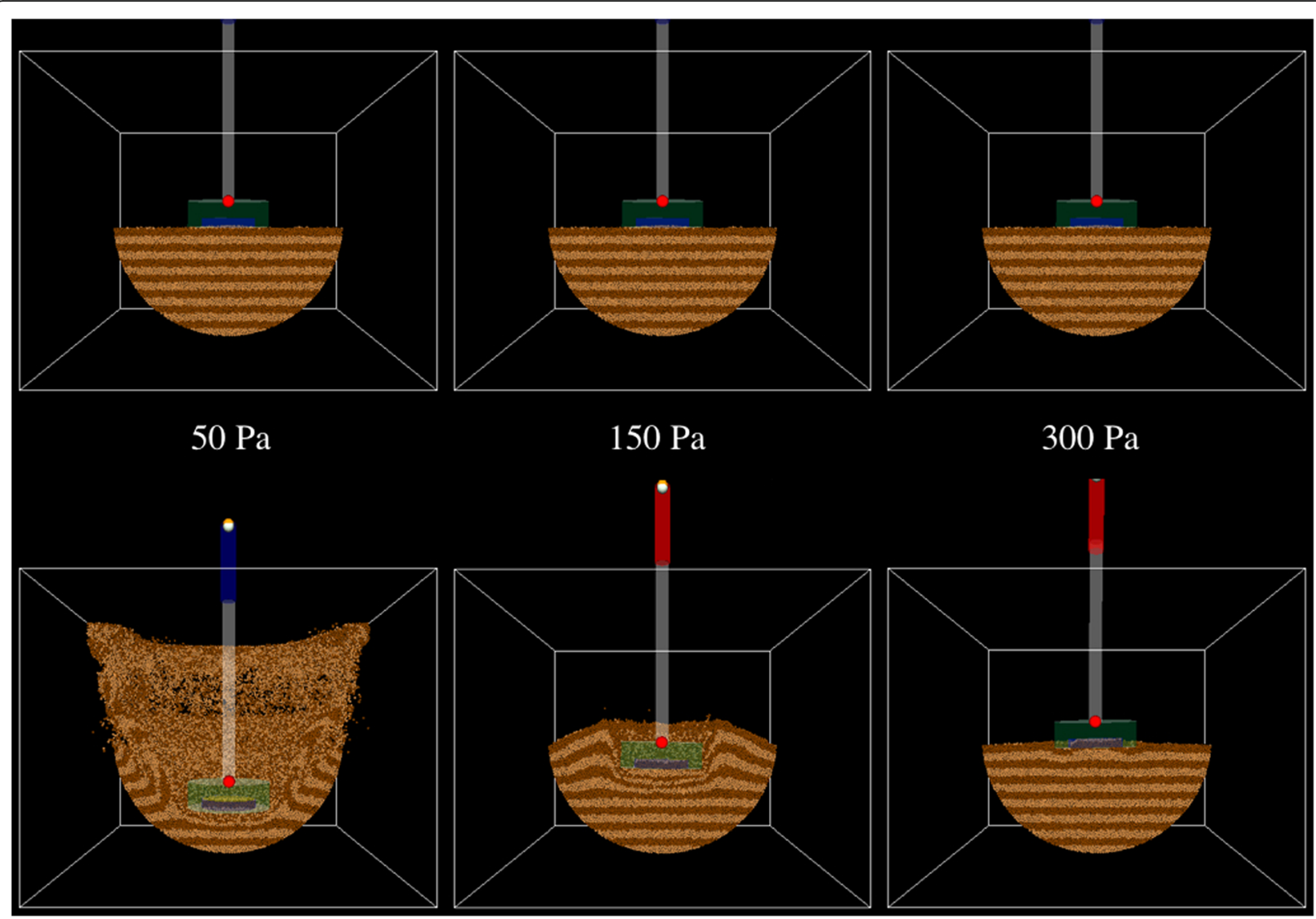

Fig. 1 Effect of regolith cohesive strength on simulated sampling encounters, modeled on the OSIRIS-REx mission. Weak regolith only offers weak resistance to the slow speed impact, while stronger regolith effectively stops penetration at the surface. The top figures show the configuration at impact, while the bottom figures show the maximum penetration. Simulation details and models are defined and discussed in Bierhaus et al. (2018)

interact gravitationally and potentially form a binary system (Jacobson and Scheeres 2011, Walsh and Jacobson 2015). For a system with $\Gamma>1$, any components separated from the surface or any fissioned components are immediately on escape trajectories and will depart from each other without any opportunities for further interactions (Scheeres 2018). Thus, transition across the parameter value $\Gamma=1$ demarcates an important shift in body evolution, making it interesting to discuss how a specific rubble pile could evolve over time to achieve this transition. Note that if the body fissions into components that are more equal in size, the escape limit occurs at values of $\Gamma$ less than unity; however, again, the simple model of failure should be revisited in this case as well.

The parameter $\Gamma$ depends on the strength of the rubble pile's matrix, its bulk density, and its total size. The matrix strength and density are expected to be homogeneous across any portion of the rubble pile and thus would be conserved when a body fissions into multiple components. Thus, only if the total size of the system changes will this parameter evolve. One way in which this can happen is through the natural evolution of a rubble pile system that undergoes fission and forms a binary system, contact binary, or asteroid pair. In any of these processes, the system will naturally lose some material through a number of ejection events, as documented in Jacobson and Scheeres (2011). If the system forms an asteroid pair (Vokrouhlicky and Nesvorny 2008, Pravec et al. 2010), then the total size of each component decreases significantly. If the system instead forms a binary or reimpacts to form a contact binary, there will still likely be some material removed from the system, decreasing its overall size. For systems trapped in a binary state, there are a variety of evolutionary mechanisms that can continue to evolve the system into a final state where the components escape, impact, or is frozen as a binary into a stable state (Walsh and Jacobson 2015). Thus, it may be natural for many rubble pile bodies to slowly evolve to smaller sizes over time, eventually reaching a parameter value of $\Gamma \sim 1$ at which point the system transitions to a different evolutionary mechanism recently studied (Scheeres 2018). 


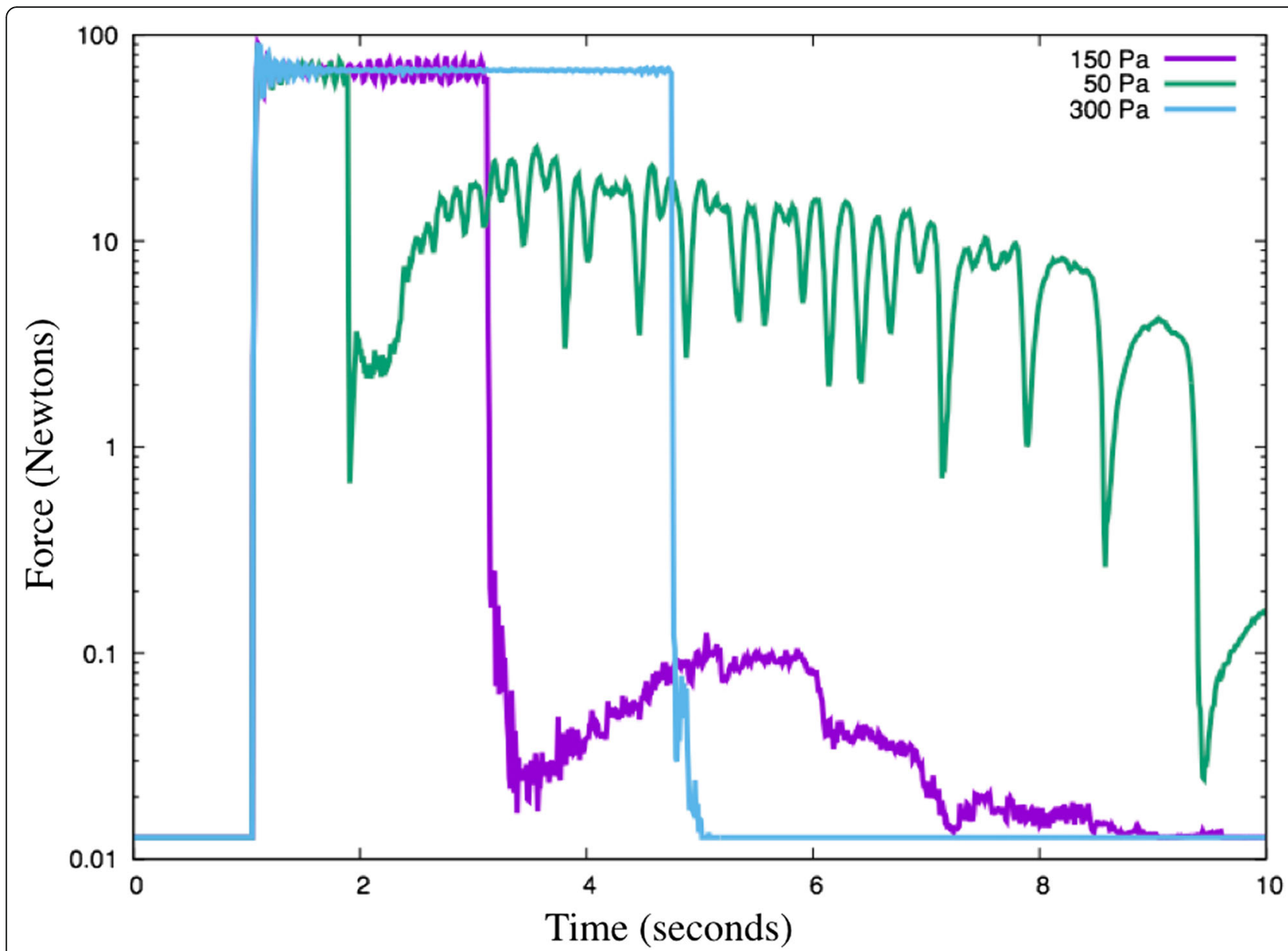

Fig. 2 Force profile acting on system center of mass for Fig. 1. The constant force segment occurs due to the constant force spring in the arm, described in Bierhaus et al. (2018)

Once this parameter value is achieved, the system can no longer form binary systems-even temporarily-and enters a phase where the body should eventually completely disaggregate into its component constituents. This occurs after every fission event the system separates into smaller components, each component escapes, and due to its smaller size can be spun up to fission at an even faster rate. The timescales for these events form a convergent power series, meaning that the disaggregation happens in a finite time. This time is, at its shortest, equal to the YORP timescale of an initial rubble pile asteroid of a given radius. Using this theory to understand the distribution of binary asteroid sizes (see Fig. 3), we see that this drop-off in binaries occurs for strengths between 1 and $100 \mathrm{~Pa}$. As we note later, however, this is within a factor of a few of inferred global strength, indicating that a more detailed analysis of the fission conditions of rubble pile bodies should be performed, improving the approximate model given in Eq. 1.

\section{Measuring strength in regolith and rubble piles}

Given the theory of cohesive regolith and having explored some of the implications, we now consider what sort of measurements can be made to verify the theory and evaluate the actual strength levels. There are two approaches to this to directly measure the strength of regolith on an asteroid or to interpret astronomical observations of bodies that imply a certain level of strength. In the following, we discuss both approaches and future prospects for the applications of these proposed approaches. In the following, we make the tacit assumption that measuring the strength of a body's regolith should be equivalent to determining its global cohesive strength. This assumption relies on our model, so that the disparate values of strength between these components would potentially invalidate the theory as given. Conversely, similar values of strength for surface regolith and global strength would support the theory and could be viewed as a prediction of the theory. 


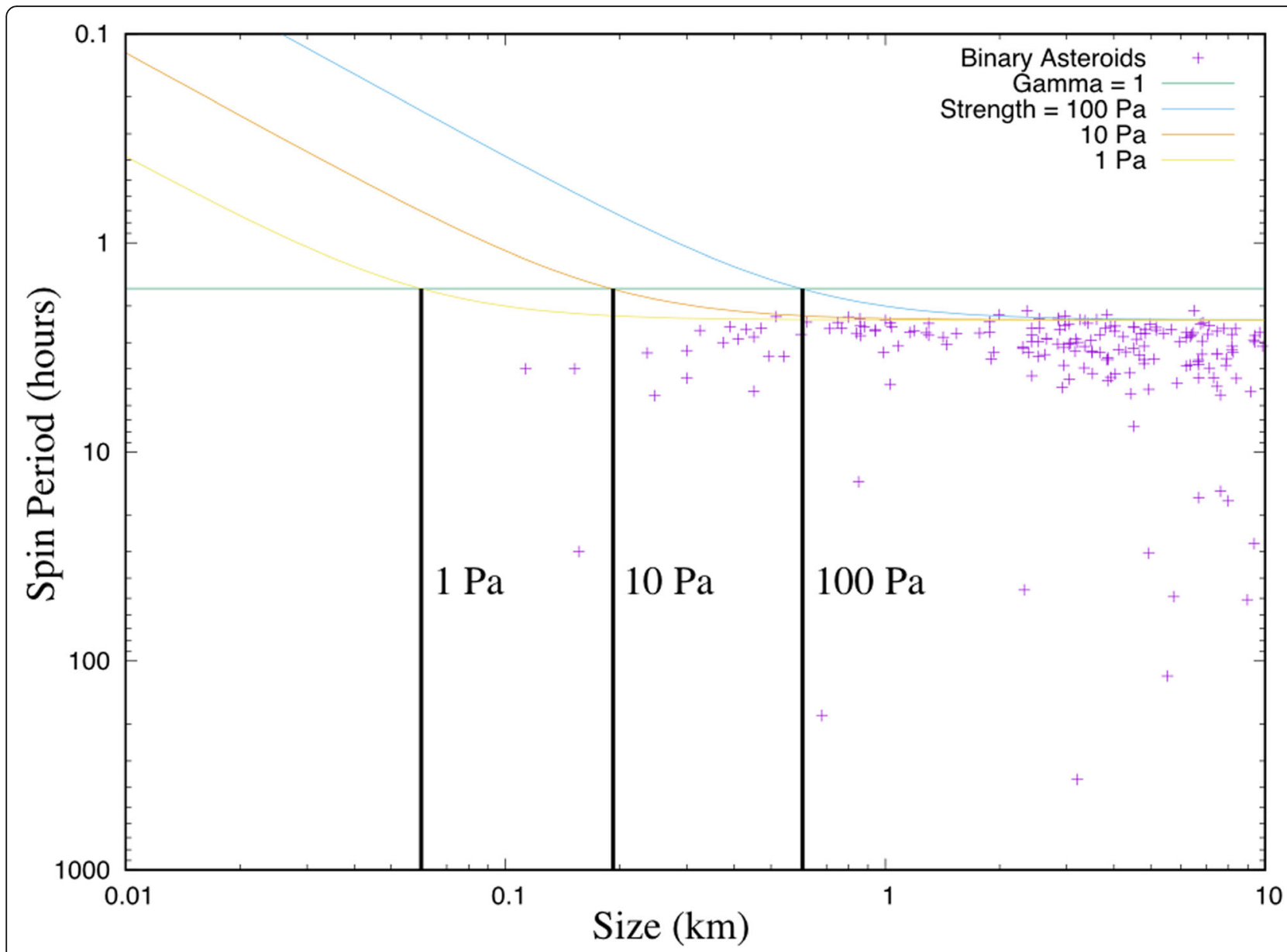

Fig. 3 Observed binary asteroid primary spin periods vs estimated diameters. Data taken from Warner et al. (2009). The vertical lines indicate where different strength levels cross the $\Gamma=1$ line, which demarcates the ideal transition between the binary formation and abrupt binary escape following fission

\section{Measurement of regolith strength from cratering}

The theory of impact and explosive cratering has been highly developed and refined in the past few decades (Holsapple 1993). Although research is still ongoing on this topic, many of the fundamental relationships between impactor or explosive energy and the resulting crater size, speed profiles, excavation time, and other resultants are now well known.

If an energetic impact or explosion of known energy occurs on the surface of a small body, it is possible, utilizing crater scaling theory, to determine the strength of regolith by observing the crater size and its formation characteristics. In the following, we will apply results of these analyses to develop a methodology for such an approach. The major question with regard to cratering theory is whether the crater forms in the gravity or the strength regime. In the gravity regime, the crater forms while working against gravity, which controls its eventual size and formation time. Conversely, in the strength regime, the crater expends most of its energy in breaking up the material. A relevant question is in what regime an energetic event on the surface of an asteroid would fall into, given the simultaneous weak strength and weak gravity. Holsapple (1993) provides a criterion for this, comparing material strength with lithostatic pressure, to find an approximate dividing line, expressed as $\sigma_{\mathrm{c}}=\rho g a$ where $\sigma_{\mathrm{c}}$ is taken as the strength of the surface regolith, $\rho$ is the regolith bulk density, $g$ is the net surface acceleration, and $a$ is a characteristic length-usually the radius of the impactor or the radius of the explosive charge. If the strength is larger than this balance, then the crater lies in the strength regime, while if it is smaller, it lies in the gravity regime. The cutoff is not sharp, however, and is only definite an order of magnitude away from this balance point. For our model density of $2000 \mathrm{~kg} / \mathrm{m}^{3}$ and surface accelerations from milli- to micro-G's, we have a ratio of strength to impactor size of $20 \rightarrow 0.02$, and for an impactor or explosive of size $10 \mathrm{~cm}$, the transition strength is $2 \rightarrow 0.002 \mathrm{~Pa}$. Thus, only for the largest gravity and weakest cohesive regolith (milli-G gravity with a 
regolith strength on the order of $1 \mathrm{~Pa}$ ) would a cratering event be at the transition point, and for most of the strengths and typical surface accelerations, we are clearly within the strength regime for cratering. Based on this computation, we will only apply the strength regime results in the following.

In the strength regime, we can use the following results that relate crater size (radius $R$ ) and formation time ( $T$ ) to the impactor energy and the surface strength, taken from Holsapple (1993) and Housen et al. (1983).

$$
\begin{aligned}
R & =\left(\frac{m}{\rho}\right)^{1 / 3}\left(\frac{\rho U^{2}}{\sigma_{\mathrm{c}}}\right)^{\mu / 2} \\
T & =\sqrt{\frac{\rho}{\sigma_{\mathrm{c}}}} R
\end{aligned}
$$

where $m$ is the impactor mass, $U$ is the impactor speed, and $1 / 3 \leq \mu \leq 2 / 3$. The parameter $\mu$ is a material property, and a typical value for sand is $1 / 2$. If instead the energy source is a surface charge of mass $W$ and specific energy $Q$, the crater radius relationship changes to (Holsapple 1993)

$$
R=\left(\frac{W}{\rho}\right)^{1 / 3}\left(\frac{\rho 2 Q}{\sigma_{\mathrm{c}}}\right)^{\mu / 2}
$$

The accuracy of this formula has been well established and can be used reliably to infer target material strength as a function of radius size and time evolution. In the following, two scenarios are investigated, interpretation of the Hayabusa2 cratering event and estimates for the proposed BASiX blast experiments.

Hayabusa2 impact crater size During the Hayabusa 2 mission, an impactor will be shot into the asteroid surface. The nominal mass of the impactor is $2 \mathrm{~kg}$, and the impact speed is $2 \mathrm{~km} / \mathrm{s}$ (Saiki et al. 2013). If we take the body's regolith to be $1250 \mathrm{~kg} / \mathrm{m}^{3}$, commensurate with the bulk density of Bennu, the target of the OSIRIS-REx mission, and a similarly primitive asteroid, the resulting crater size will be $R_{\mathrm{H} 2}=31 / \sigma_{\mathrm{c}}^{1 / 4}$, and for our range of strengths from 10 to $100 \mathrm{~Pa}$ will yield a crater radius of size $17 \rightarrow 9.3 \mathrm{~m}$ (from 10 to $100 \mathrm{~Pa}$ ), larger than the mission stated value of $2 \mathrm{~m}$ (Saiki et al. 2013) which would correspond to a strength of almost $60 \mathrm{kPa}$, more appropriate for impact into a coherent (if weak) rock. The predicted formation time is $T_{\mathrm{H} 2}=18 / \sigma_{\mathrm{c}}^{3 / 4} \mathrm{~min}$, with a corresponding range of $3.2 \rightarrow 0.6 \mathrm{~min}$. Unfortunately, it is not clear whether the crater formation time will be observed during the impact, which removes this one important constraint.

BASiX blast crater size A different approach has been proposed in the discovery mission proposal BASiX
(Anderson et al. 2014). In that mission proposal, a $5 \mathrm{~kg}$ mass of explosives with $Q=4 \mathrm{MJ} / \mathrm{kg}$ is to be placed on an asteroid's surface and detonated (remotely). Applying Eqs. 2 and 3, again, the resulting crater radius is $R_{\mathrm{B}}=50 / \sigma_{\mathrm{c}}^{1 / 4}$ with a range $28 \rightarrow 15 \mathrm{~m}$ (from 10 to $100 \mathrm{~Pa}$ ). The time evolution is $T_{\mathrm{B}}=30 / \sigma_{\mathrm{c}}^{3 / 4}$ with a range $5.3 \rightarrow 0.8$ minutes.

Strength measurement relative accuracy Given these example computations, we can consider how measurements of crater size and formation time can be inverted to estimate the strength of regolith. First, we assume that the other information on density, impactor or blast mass and energy, and parameter $\mu$ has been determined. Then, if a crater can be measured with an accuracy of $\delta R$, the inferred strength from Eq. 2 can be measured to an accuracy $\delta \sigma_{\mathrm{c}} / \sigma_{\mathrm{c}}=4 \delta R / R$. Similarly, if the crater excavation time can be estimated to an accuracy of $\delta T$, the inferred strength from Eq. 3 can be measured to an accuracy $\delta \sigma_{\mathrm{c}} / \sigma_{\mathrm{c}} \sim 0.75 \delta T / T$. Ultimately, as the crater size measurement is static, the crater radius observation should yield a greater precision for use in inferring the regolith strength.

The main uncertain parameter in Eqs. 2 and 3 beyond the measurement of the radius or formation time is the parameter $\mu$. If we take $\mu=1 / 2$, then the range of values it can take on is $\delta \mu= \pm 1 / 6$; however, the resultant crater sizes across this variation are quite large. This indicates the need to measure this key quantity to better interpret the resulting information. Here, we can rely on comparisons with material with similar morphology for terrestrial tests and on mechanical property tests of samples returned from the Hayabusa2 and OSIRIS-REx missions.

\section{Measurement of rubble pile strength from astronomical observations}

There are a few astronomical observations that have also provided estimates of the bulk strength of cohesive asteroids. Specifically, there are three different asteroid observations that relate to this, the active asteroid 2013 P/R3, asteroid 1950 DA, and 2008 TC3 (the Almahatta Sitta meteorite). These are discussed in turn in the following. Taken together, they provide estimates of global strength on the order of $10-100 \mathrm{~Pa}$, consistent with what is predicted from our model. They are not definitive, as they are not direct determinations of strength; however, they do provide insight into what the structure of rubble pile bodies may be.

Active asteroid 2013 P/R3 Jewitt et al. (2014) report on a series of observations of what initially was classified as a cometary body, due to an observed coma. Subsequent observations showed that this body was actually 


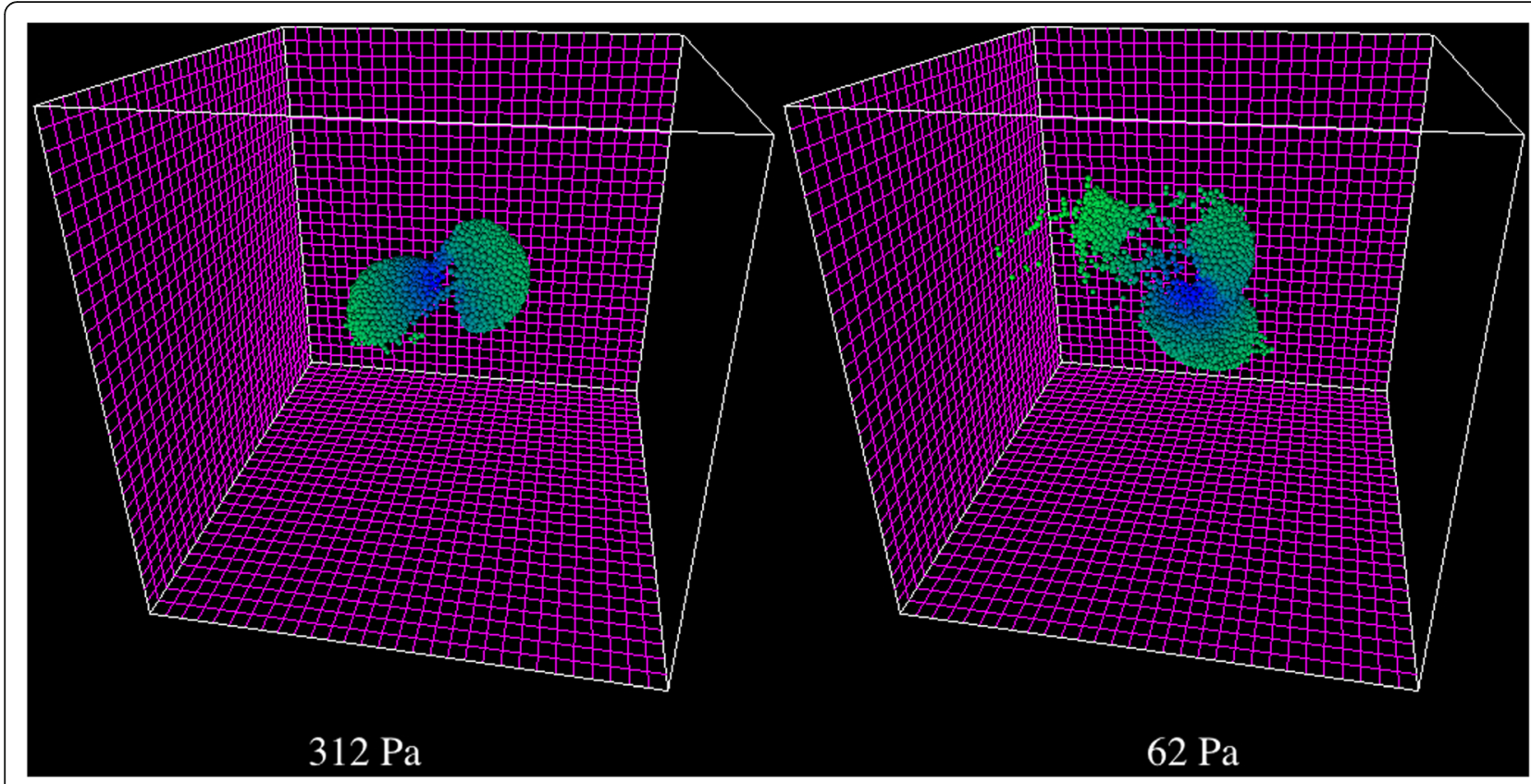

Fig. 4 Asteroid fission simulations, showing the effect of different levels of cohesion on the morphology of how the body splits. Figures taken from Sánchez and Scheeres (2016)

undergoing a disruption process, separating into several distinct clusters, each of which was in turn comprised of smaller clusters. In addition, the original coma was more clearly identified as a size distribution of dust and grains from this disrupted system. Jewitt et al. (2014) considered a number of possible formation scenarios, including impact disruption and rotational fission.

Using the observed relative motion between the largest components of this system and assuming a rotational fission event (see Fig. 4 for a representation of how such a failure may appear), Hirabayashi et al. (2014) analyzed the necessary strength of the original body. Taking estimates of the total size of the original component, the relative speed between the components, and a range of possible densities, they found that this original system would have needed a global strength between 40 and $210 \mathrm{~Pa}$ to create the observed system. More recently, Jewitt et al. (2017) reprocessed the observations to narrow the range of uncertain parameters and, by adding additional constraints on the spectral type of the body, were able to narrow the strength range of the initial system to 50-100 Pa. We note that this is a valuable upper limit on the strength, since it is inferred from a body that has broken up.

Asteroid 1950 DA Asteroid 1950 DA is an NEA that has been observed with astrometry, spectroscopy, photometry, and using resolved range-Doppler radar imaging. Resulting from these observations are a very precise set of constraints on this object, including an estimated size, density, and shape (Busch et al. 2007). Combining all of these observations, Rozitis et al. (2014) were able to determine that this body was spinning beyond its expected cohesionless gravitational reshaping limit, indicating that it requires a global strength of at least $60 \mathrm{~Pa}$ to hold together. A more detailed geophysical model by Hirabayashi and Scheeres (2015) was able to probe the needed global strength for this body assuming a homogeneous strength distribution and its relatively precise shape model and found a minimum strength of $75-85 \mathrm{~Pa}$ as a function of body density. It should be noted that the failure condition and associated spin rate changes for different internal distributions of strength and thus that these limits are inherently uncertain, but that the overall order of magnitude of the failure strength should be accurate. Unlike the previous case, this is a lower bound on strength as the body has not been seen to disrupt. As the asteroid is relatively large and has a distended equatorial region, it is reasonable to assume that it has undergone some degree of reshaping due to its rapid spin rate. This would indicate that the determined strength may be close to its actual strength.

Asteroid 2008 TC3, the Almahatta Sitta meteorite As a final case, we consider the asteroid 2008 TC3, which was observed prior to entry into the Earth's atmosphere and which fell over Sudan, being recovered as the Almahatta Sitta meteorite (Jenniskens et al. 2009). While not a definitive case, for reasons detailed below, this also provides some possible insight into the structure of small rubble pile bodies. This particular body is of interest as its meteorite fall was comprised of a wide variety of components of 
different compositions, indicating that the original body was comprised of several different parent bodies.

Pre-entry observations of the asteroid showed that it was at most $5 \mathrm{~m}$ in diameter and was observed to be tumbling with a spin rate of around 3 min (Scheirich et al. 2010), which from our analysis would only require a strength on the order of $25 \mathrm{~Pa}$ to hold together as a rubble pile. Observations of the initial trajectory also indicated significant macroporosity (Kohout et al. 2011) and a high breakup altitude, indicating a "weak" body (Popova et al. 2011). In addition, substantial loss of $1-10 \mu \mathrm{m}$ material was observed in the upper atmosphere (Borovicka and Charvat 2009). All of these components are consistent with the body being a cohesive rubble pile. Further, the fact that it was tumbling also fits with the proposed evolutionary model for the disaggregation of rubble pile bodies outlined in Scheeres (2018).

A significant caveat exists with this observation, however. In Borovicka (2015), it is noted that the breakup conditions for this asteroid were determined to have occurred at a dynamic pressure of $50 \mathrm{kPa}$ in the upper atmosphere-much stronger than the strengths discussed here. The analysis of meteor breakup strength using dynamic pressure as a proxy is not fully understood, however, and more detailed simulations of breakup mechanics show that even a strengthless body will break up at a non-zero level of dynamical pressure (Svetsov 1995). This points out a larger issue related to uncertainties in the modeling of meteor breakup in the upper atmosphere whose resolution could better enable the correlation of meteorite strength with breakup altitude (Register et al. 2017).

\section{Discussion}

The consistency of rubble pile global strength measurements with predictions from the regolith strength theory are interesting and provocative, although they do not establish that the theory as outlined herein is in fact true. Thus, it is of interest to consider how the theory of regolith strength and global rubble pile strength could be established based on disparate measurements of regolith strength and on observations of global strength of bodies. The initial step would be to show the consistency of the theory with observed regolith strength. The Hayabusa2 and OSIRIS-REx missions provide the next opportunity to observe a primitive body, and each of these missions plans on some interactions with the surface that could provide insight. The Hayabusa2 interaction with the impact cratering event provides the best test of regolith strength, and results from that experiment will need to be interpreted in consideration of the other observed properties of the asteroid and of the impact site. Important considerations include whether the site consists of boulders or of finer regolith, other observations on the surface morphology of the asteroid to determine the properties of regolith on the body, and global properties such as bulk density. The OSIRIS-REx mission provides a much more limited opportunity to constrain the regolith strength, based on the surface interaction between the sampling arm and the surface. This interaction is quite brief and will be interrupted by the release of gas, whose interaction with the regolith will be interesting but difficult to fully interpret given the lack of instrumentation to measure relevant effects.

While there are always future proposed missions designed to probe such questions, that cannot be the basis for current evaluations of this possible effect. Thus, it is also of interest to reexamine the NEAR and Hayabusa observations of the asteroids Eros and Itokawa to see if further information can be gleaned from them. Possible implications of regolith strength include the efficiency of resurfacing on these bodies, the angles of repose observed in regions of high slope, and the interpretation of high-resolution imagery. There has only been a limited study of some of these effects. One notable example is the seismic shaking analysis by Thomas and Robinson (2005). Future work could reexamine the descent images that NEAR took of Eros and the close images that Hayabusa took of Itokawa. The question of interest includes limits on the burial depth of boulders, the depth of features seen on the surface, and other interpretations. These specific analyses are beyond the current paper, but hopefully could be motivated by it.

\section{Conclusions}

This paper reviews and applies the Sánchez and Scheeres (2014) theory of regolith strength to global and surface analysis of asteroids. Beyond discussing the implications of these effects, collected together here for the first time, the paper also proposes ways in which surface regolith strength could be measured in the future. Global measures of rubble pile strength have also been compared with predictions from the theory, with consistent values being noted. Finally, a discussion placing these comparisons into context is given. Understanding the strength of rubble pile asteroids is crucial to our understanding of their lifetime and evolution in the solar system.

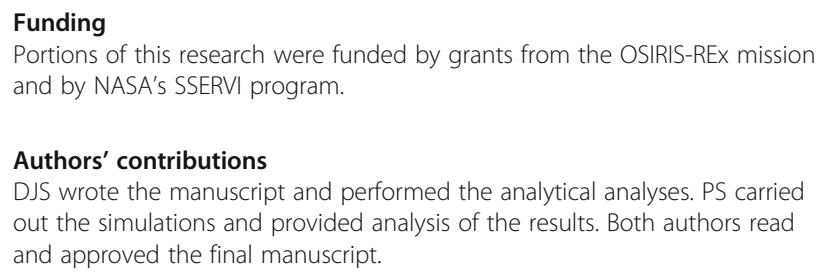

Authors' contributions

DJS wrote the manuscript and performed the analytical analyses. PS carried out the simulations and provided analysis of the results. Both authors read and approved the final manuscript.

Competing interests

The authors declare that they have no competing interests.

\section{Publisher's Note}

Springer Nature remains neutral with regard to jurisdictional claims in published maps and institutional affiliations. 
Received: 1 December 2017 Accepted: 9 April 2018

Published online: 02 May 2018

\section{References}

Anderson RC, Scheeres D, Chesley S, BASiX Team (2014) Binary asteroid in-situ explorer mission (BASiX): a mission concept to explore a binary near earth asteroid system. Lunar Planet Sci Confer 45:1571

Asphaug El (2009) Shattered dirt: surface fracture of granular asteroids. Lunar Planet Sci 40 Abstract \#1438

Bierhaus EB, Britt D, Clark B, Hartzell CM, Gertsch L, Kulchitsky AV, Johnson JB, Metzger P, Reeves DM, Sánchez P, Scheeres DJ, Zacny K (2018) In: Abreu NM (ed) Geotechnical properties of asteroids affecting surface operations, mining and ISRU activities, in primitive meteorites and asteroids. Tucson: Elsevier in press

Borovicka J (2015) About the definition of meteoroid, asteroid, and related terms. IAU Gen Assemb 22

Borovicka J, Charvat Z (2009) Meteosat observation of the atmospheric entry of 2008 TC3 over Sudan and the associated dust cloud. Astro Astrophys 507(2):1015-1022

Busch MW, Giorgini JD, Ostro SJ, Benner LAM, Jurgens RF, Rose R, Hicks MD, Pravec P, Kusnirak P, Ireland MJ, Scheeres DJ, Broschart SB, Magri C, Nolan MC, Hine AA, Margot JL (2007) Physical modeling of near-Earth asteroid (29075) 1950 DA. Icarus 190(2):608-621

Castellanos A (2005) The relationship between attractive interparticle forces and bulk behaviour in dry and uncharged fine powders. Adv Phys 54(4):263-376

Hirabayashi M, Scheeres DJ (2015) Stress and failure analysis of rapidly rotating asteroid (29075) 1950 DA. Astrophys J L 798:L8

Hirabayashi M, Scheeres DJ, Sánchez P, Gabriel T (2014) Constraints on the physical properties of Main Belt Comet P/2013 R3 from its breakup event. Astrophys J Lett 789:L12 (5pp)

Holsapple KA (1993) The scaling of impact processes in planetary sciences. Annu Rev Earth Planet Sci 21(1):333-373

Holsapple KA (2010) On YORP-induced spin deformations of asteroids. Icarus 205(2):430-442

Housen KR, Schmidt RM, Holsapple KA (1983) Crater ejecta scaling laws: fundamental forms based on dimensional analysis. J Geophys Res Solid Earth 88(B3):2485-2499

Jacobson SA, Scheeres DJ (2011) Dynamics of rotationally fissioned asteroids: source of observed small asteroid systems. Icarus 214(1):161-178

Jenniskens P, Shaddad MH, Numan D, Elsir S, Zolensky ME KAM, Le L, Robinson GA, Friedrich JM, Rumble D et al (2009) The impact and recovery of asteroid 2008 TC3. Nature 458(7237):485-488

Jewitt D, Agarwal J, Li J, Weaver H, Mutchler M, Larson S (2014) Disintegrating asteroid P/2013 R3. Astrophys J Lett 784(1):L8

Jewitt D, Agarwal J, Li J, Weaver H, Mutchler M, Larson S (2017) Anatomy of an asteroid breakup: the case of P/2013 R3. Astron J 153(5):223

Kohout T, Kiuru R, Montonen M, Scheirich P, Britt D, Macke R, Consolmagno G (2011) Internal structure and physical properties of the asteroid 2008 TC 3 inferred from a study of the Almahata Sitta meteorites. Icarus 212(2):697-700

Lauretta DS, Balram-Knutson SS, Beshore E, Boynton WV, d'Aubigny CD, DellaGiustina DN, Enos HL, Gholish DR, Hergenrother CW, Howell ES et al (2017) OSIRIS-REx: sample return from asteroid (101955) Bennu. Space Sci Rev in press

Michikami T, Nakamura AM, Hirata N, Gaskell RW, Nakamura R, Honda T, Honda C, Hiraoka K, Saito J, Demura H et al (2008) Size-frequency statistics of boulders on global surface of asteroid 25143 Itokawa. Earth Planets Space 60(1):13-20

Perko HA, Nelson JD, Sadeh WZ (2001) Surface cleanliness effect on lunar soil shear strength. J Geotech Geoenviron Eng 127(4):371-383

Popova O, Borovicka J, Hartmann WK, Spurny P, Gnos E, Nemtchinov I, TrigoRodriguez JM (2011) Very low strengths of interplanetary meteoroids and small asteroids. Meteorit Planet Sci 46(10):1525-1550

Pravec P, Vokrouhlicky D, Polishook D, Scheeres DJ, Harris AW, Galad A, Vaduvescu O, Pozo F, Barr A, Longa P, Vachier F, Colas F, Pray DP, Pollock J, Reichart D, Ivarsen K, Haislip J, LaCluyze A, Kusnirak P, Henych T, Marchis F, Macomber B, Jacobson SA, Krugly YN, Sergeev A, Leroy A (2010) Formation of asteroid pairs by rotational fission. Nature 466:1085-1088

Register PJ, Mathias DL, Wheeler LF (2017) Asteroid fragmentation approaches for modeling atmospheric energy deposition. Icarus 284:157-166

Rozitis B, MacLennan E, Emery JP (2014) Cohesive forces prevent the rotational breakup of rubble-pile asteroid (29075) 1950 DA. Nature 512:174
Saiki T, Sawada H, Okamoto C, Yano H, Takagi Y, Akahoshi Y, Yoshikawa M (2013) Small carry-on impactor of Hayabusa2 mission. Acta Astronautica 84:227-236

Sánchez P, Scheeres DJ (2012) DEM simulation of rotation-induced reshaping and disruption of rubble-pile asteroids. Icarus 218:876-894

Sánchez P, Scheeres DJ (2014) The strength of regolith and rubble pile asteroids. Meteorit Planet Sci 49(5):788-811

Sánchez P, Scheeres DJ (2016) Disruption Patterns of Rotating Self-Gravitating Aggregates: A Survey on Angle of Friction and Tensile Strength. Icarus 271: 453-471

Scheeres DJ (2018) Disaggregation of small, cohesive rubble pile asteroids due to YORP. Icarus 304:183-191

Scheeres DJ, Hartzell CM, Sánchez P, Swift M (2010) Scaling forces to asteroid surfaces: the role of cohesion. Icarus 210:968-984

Scheirich P, Durech J, Pravec P, Kozubal M, Dantowitz R, Kaasalainen M, Betzler AS, Beltrame P, Muler G, Birtwhistle P et al (2010) The shape and rotation of asteroid 2008 TC3. Meteorit Planet Sci 45(10-11):1804-1811

Svetsov W, Nemtchinov IV, Teterev AV (1995) Disintegration of large meteoroids in Earth's atmosphere: theoretical models. Icarus 116(1):131-153

Thomas PC, Robinson MS (2005) Seismic resurfacing by a single impact on the asteroid 433 Eros. Nature 436(7049):366-369

Tsuchiyama A, Uesugi M, Matsushima T, Michikami T, Kadono T, Nakamura T, Uesugi K, Nakano T, Sandford SA, Noguchi R, Matsumoto T, Matsuno J, Nagano T, Imai Y, Takeuchi A, Suzuki Y, Ogami T, Katagiri J, Ebihara M, Ireland TR, Kitajima F, Nagao K, Naraoka H, Noguchi T, Okazaki R, Yurimoto H, Zolensky ME, Mukai T, Abe M, Yada T, Fujimura A, Yoshikawa M, Kawaguchi J (2011) Three-dimensional structure of Hayabusa samples: origin and evolution of Itokawa regolith. Science 333:1125-1128

Vokrouhlicky D, Nesvorny D (2008) Pairs of asteroids probably of a common origin. Astron J 136(1):280

Walsh K, Jacobson SA (2015) Formation and evolution of binary asteroids. Asteroids IV, 375-393. University of Arizona Press, Tucson

Wang X, Schwan J, Hsu H-W, Grun E, Horanyi M (2016) Dust charging and transport on airless planetary bodies. Geophys Res Lett 43(12):6103-6110

Warner BD, Harris AW, Pravec P (2009) The asteroid lightcurve database. Icarus 202(1):134-146

Yoshikawa M, Watanabe S, Tsuda Y, Kuninaka H et al (2014) Hayabusa2-the next asteroid sample return mission of Japan. Transact Jpn Soc Aeronaut Space Sci 12(29):29-33

\section{Submit your manuscript to a SpringerOpen ${ }^{\circ}$ journal and benefit from:}

- Convenient online submission

- Rigorous peer review

- Open access: articles freely available online

- High visibility within the field

- Retaining the copyright to your article

Submit your next manuscript at $\gg$ springeropen.com 